\title{
Characterization of selective exosomal microRNA expression profile derived from laryngeal squamous cell carcinoma detected by next generation sequencing
}

\author{
QIANG HUANG, JIECHAO YANG, JUAN ZHENG, CHIYAO HSUEH, YANG GUO and LIANG ZHOU \\ Department of Otorhinolaryngology Head and Neck Surgery, Eye, Ear, Nose, and Throat Hospital, \\ Fudan University, Shanghai 200031, P.R. China
}

Received March 8, 2018; Accepted August 13, 2018

DOI: $10.3892 /$ or.2018.6672

\begin{abstract}
Exosomes are nanometer-scale extracellular vesicles derived from almost all types of cells and key signaling mediators between cancer cells and their microenvironment. Certain microRNAs (miRNAs) are selected for exosome packing and exclusion from parental cells, while other miRNAs are selectively retained by cells, suggesting a biological role for these miRNAs in tumor malignant progression. In the present study, we isolated and characterized the exosomes derived from the laryngeal squamous cell carcinoma (LSCC) cell line AMC-HN-8 for the first time, and identified a subset of miRNAs enriched in the exosomes compared with parental cells, such as miR-1246, miR-1290, miR-335-5p, miR-127-3p and miR-122-5p through small RNA sequencing combined with reverse transcription-quantitative PCR (RT-qPCR) analysis. Potential target prediction, Gene Ontology (GO) annotation and Kyoto Encyclopedia of Genes and Genomes (KEGG) pathway enrichment analysis revealed possible functions associated with these selective exosomal miRNAs. In conclusion, the present study demonstrated that the LSCC cell line AMC-HN-8 can release exosomes and cells can selectively pack certain miRNAs into exosomes.
\end{abstract}

Correspondence to: Dr Liang Zhou, Department of Otorhinolaryngology Head and Neck Surgery, Eye, Ear, Nose, and Throat Hospital, Fudan University, Building 10, Room 307, 83 Fenyang Road, Shanghai 200031, P.R. China

E-mail: zhoulent@126.com

Abbreviations: miRNA, microRNA; LSCC, laryngeal squamous cell carcinoma; RT-qPCR, reverse transcription quantitative PCR; GO, Gene Ontology; KEGG, Kyoto Encyclopedia of Genes and Genomes; TEM, transmission electron microscope; NTA, nanoparticle tracking analysis; FCM, flow cytometry

Key words: laryngeal squamous cell carcinoma, exosomes, microRNAs, selective expression, head and neck carcinoma

\section{Introduction}

Laryngeal squamous cell carcinoma (LSCC) remains one of the most common cancers of the upper respiratory tract. Even though advanced treatment options are available, including surgery, chemotherapy and radiotherapy, mortality associated with LSCC is still extremely high (1). Statistics have revealed that LSCC is one of a few cancers in which the 5 -year survival rate has decreased from 66 to $63 \%$ over the past 40 years, while the overall incidence is decreasing (2). Therefore, it is urgent to identify specific biomarkers that can be used in the early diagnosis and prognosis of LSCC. Since miRNAs have been reported to be present in the blood of cancer patients (3), utilized for targeted cancer therapy (4) and used in cancer prognosis (5), it is universally acknowledged that deregulated miRNA expression has an important effect on cellular genes which regulate the proliferation, metastasis and the cell cycle, leading to the progression of $\operatorname{LSCC}(6,7)$. Notably, due to the inherent heterogeneity of the miRNA sources in the blood and tendency to degrade, free miRNA in the blood is not an ideal biomarker in the diagnosis and prognosis of LSCC.

Extracellular vesicles, particularly exosomes, which are membrane-bound particles ranging from 30-150 nm and not merely lipid vesicles, also carry cargoes including nucleic acids, proteins, receptors, lipids, and transcription factors (8). Exosomes are present in biological fluids and are involved in multiple pathological and physiological processes (9). They can either be picked up by adjacent cells or carried to distant sites via biological fluids and may therefore induce phenotypic modifications in recipient cells (10). They are promising candidates in the field of tumor liquid biopsy on account of cancer-specific expression profiles and reflect cancer-bearing status. To date, studies focusing on exosomal miRNAs indicated that exosomal miRNAs are of value for diagnostic and prognostic significance in head and neck carcinoma, including nasopharyngeal, oral squamous cell carcinoma and LSCC. Previous studies indicated that exosomal miR-24-3p impeded T-cell function by targeting fibroblast growth factor (FGF11) and may serve as a potential prognostic biomarker for nasopharyngeal carcinoma (11). Li et al demonstrated that exosomes derived from hypoxic oral squamous cell carcinoma 
cells delivered miR-21 to normoxic cells to elicit a prometastatic phenotype (12). Additionally, Wang et al found that serum exosomal miR-21 along with HOTAIR were significantly correlated with clinical parameters of LSCC (13). These findings indicate that exosomal miRNAs have a distinctly important effect on the malignant progression of head and neck carcinoma.

Exosomes contain selected miRNAs that could contribute to intercellular communication (14). The process by which several miRNAs are enclosed in exosomes is selective rather than indiscriminate $(15,16)$. Despite growing interest in studying the exosomal miRNA difference between cancer cells and normal cells, we still lack an understanding of the difference between parental cellular miRNA and exosomal miRNA. Honegger et al presented the first comprehensive analysis of cellular and exosomal miRNAs, suggesting that there exists an enormous difference between them (17). To the best of our knowledge, the distribution characteristics and comprehensive expression profile on the RNA content of LSCC-derived exosomes remains unknown.

The overall goal of this study was to identify and characterize selective exosomal miRNA expression profiles and speculate their potential target via bioinformatics analysis. To achieve this objective, we first isolated the exosomes derived from the LSCC cell line AMC-HN-8, and then characterized exosome pellets with transmission electron microscope (TEM), nanoparticle tracking analysis (NTA) and flow cytometry (FCM). After extraction of total RNA from cells and exosomes, next generation sequencing was carried out. Notably, we identified that miR-1246, miR-1290, miR-335-5p, miR-127-3p and miR-122-5p were upregulated and miR-4521, miR-4483, miR-30b-5p, miR-29b-3p and miR-374b-5p were downregulated in exosomes compared with parental cells Finally, we revealed the potential targets of these selective exosomal miRNAs via bioinformatics analysis. Collectively, we speculated that these selective exosomal miRNAs may play an important role in LSCC, and shed light on the biological implication of LSCC and provided a theoretical base for the further research.

\section{Materials and methods}

Cell culture and generation of exosome-depleted FBS. The human laryngeal squamous carcinoma cell line AMC-HN-8 which was established by Kim et al in 1997 (18) from patients with head and neck cancer was preserved in our laboratory. Laryngeal squamous carcinoma cell lines Tu212 and Tu686 were obtained from the Central South University (Hunan, China). All 3 cell lines are representative in vitro models for studying the biology of head and neck carcinoma. All cells were cultured in RPMI-1640 (HyClone; GE Healthcare Life Sciences, Logan, UT, USA), $1 \%$ penicillin-streptomycin (Genom Biotechnology, Hangzhou, China) and 10\% exosome-depleted fetal bovine serum (FBS; Gibco; Thermo Fisher Scientific, Inc., Waltham, MA, USA) in humidified air with $5 \% \mathrm{CO}_{2}$ at $37^{\circ} \mathrm{C}$.

The generation of exosome-depleted FBS was carried out by ultracentrifugation to reduce contamination from bovine exosomes. Briefly, centrifuge tubes were loaded with FBS and centrifuged at $120,000 \times \mathrm{g}$ for $6 \mathrm{~h}$ at $4^{\circ} \mathrm{C}$ (Beckman Coulter
Optima L-100XP Ultracentrifuge, SW 32Ti; Beckman Coulter, Inc., Fullerton, CA, USA). The supernatant of FBS was then filtered using a $0.22-\mu \mathrm{m}$ filter (Merck KGaA, Darmstadt, Germany).

Conditioned medium collection and exosome isolation. Cells were cultured in conditioned medium containing $10 \%$ exosome-depleted FBS for $72 \mathrm{~h}$ at $90-100 \%$ density. The conditioned medium was harvested and centrifuged at 2,000 $\mathrm{x}$ g for $10 \mathrm{~min}$ followed by $10,000 \mathrm{x} \mathrm{g}$ for $30 \mathrm{~min}$ at $4^{\circ} \mathrm{C}$. Then, the supernatant was filtered using a $0.22-\mu \mathrm{m}$ filter to eliminate cellular debris thoroughly and concentrated using centrifugal ultrafiltration (Amicon ${ }^{\circledR}$ Ultra-15 $100 \mathrm{KDa}$; Merck KGaA) to minimize the potential contamination, respectively.

Exosomes were isolated from processed conditioned medium with Ribo ${ }^{\mathrm{TM}}$ Exosome Isolation Reagent (Guangzhou RiboBio Co., Ltd., Guangzhou, China), according to the manufacturer's instructions. Briefly, processed conditioned medium was mixed with $\mathrm{Ribo}^{\mathrm{TM}}$ Exosome Isolation Reagent at a ratio of 3:1 and incubated at $4^{\circ} \mathrm{C}$ overnight. After centrifugation at $1,500 \mathrm{x} \mathrm{g}$ for $30 \mathrm{~min}$, exosomes pellets were resuspended in appropriate phosphate-buffered saline (PBS; HyClone; GE Healthcare Life Sciences) for TEM, NTA and further research. The exosome pellets were used immediately or stored at $-80^{\circ} \mathrm{C}$ until use.

TEM. The morphology of exosome pellets was examined by TEM. Briefly, a 20- $\mu 1$ of exosome-PBS solution drop was loaded onto carbon-coated copper grids and permitted to stand for $1 \mathrm{~min}$. The filter paper was utilized to remove the excess solution. Then the exosomes pellets were negatively stained with $20 \mu$ l uranyl acetate dihydrate (2\%) and allowed to stand for $1 \mathrm{~min}$. Subsequently, the filter paper was utilized to remove the excess fluid, again. The sample was allowed to dry under a lamp for 10 min before viewing on an FEI Tecnai G2 Spirit transmission electron microscope (FEI Company, Hillsboro, OR, USA) operated at $80 \mathrm{kV}$.

NTA and FCM. For the purpose of demonstrating the particle size distribution, NTA was performed using NanoSight NS300 (Malvern Instruments, Inc., Westborough, MA, USA), according to the operating instructions. In addition, FCM (BD Accuri C6 flow cytometer; BD Biosciences, Franklin Lakes, NJ, USA) was used to detect exosomal surface markers. Briefly, exosome pellets were resuspended in $100 \mu \mathrm{l}$ filtered PBS, and then $20 \mu \mathrm{l}$ immunofluorescence antibody CD63 (CD63-Antibody-FITC; cat. no. 557288) and CD81 (CD81-Antibody-FITC; cat. no. 551108; both from BD Biosciences) were added to the exosome-PBS solution. Following incubation, FCM was utilized to detect exosomal surface markers.

Total RNA extraction. Total RNA in cells and exosomes was extracted using TRIzol reagent (Invitrogen; Thermo Fisher Scientific, Inc., Waltham, MA, USA) as previously described (14). The quality and concentration of RNA were assessed using NanoDrop 2000 Spectrophotometer (NanoDrop Technologies; Thermo Fisher Scientific, Inc.). RNA content was assessed using an Agilent 2200 Bioanalyzer (Agilent Technologies, Inc., Santa Clara, CA, USA). Total RNA was 
sequenced or reverse-transcribed to cDNA immediately for further research.

Small RNA sequencing. Small RNA library preparation and sample sequencing were accomplished with the assistance of Guangzhou RiboBio Co., Ltd., using an Illumina HiSeq $^{\mathrm{TM}} 2500$ device. Total RNA from LSCC AMC-HN-8 cells $(n=3)$ and exosomes $(n=3)$ were concatenated with 5 - and 3 '-adaptors. After cDNA synthesis and PCR amplification, the cDNA library (18-40 nt) was obtained using an acrylamide gel purification method. Then, single end (SE) sequencing was performed: 1x50 bp.

$R T-q P C R$ validation. To validate the results of sequencing, we selected 6 miRNAs (miR-1246, miR-122-5p, miR-320d, miR-4483, miR-30c-5p and let-7e-5p) for further research by RT-qPCR in all 3 cell lines and its exosomes. Total RNA (300 ng) was reversed-transcribed using PrimeScript ${ }^{\mathrm{TM}}$ RT reagent Kit (Takara Biotechnology Co., Ltd., Dalian, China) according to the manufacturer's instructions. The expression level of miRNA was measured using SYBR ${ }^{\circledR}$ Premix Ex $\operatorname{Taq}^{\mathrm{TM}}$ (Takara Biotechnology Co., Ltd.) following the manufacturer's instructions. We used miR-93-5p as an internal control, which has a stable expression level between cells and exosomes, to normalize the relative expression ratio of miRNA, using the $2^{-\Delta \Delta \mathrm{Cq}}$ method (19). An ABI 7500 PCR system (Applied Biosystems; Thermo Fisher Scientific, Inc.) was used to perform the RT-qPCR and analyze the data. The thermocycling conditions were as follows: holding stage: $95^{\circ} \mathrm{C}$ for $30 \mathrm{sec}$; cycling stage: $95^{\circ} \mathrm{C}$ for $5 \mathrm{sec}$ and $60^{\circ} \mathrm{C}$ for $34 \mathrm{sec}$, 40 cycles; melt curve stage: $95^{\circ} \mathrm{C}$ for $15 \mathrm{sec}, 60^{\circ} \mathrm{C}$ for $1 \mathrm{~min}$ and $95^{\circ} \mathrm{C}$ for $15 \mathrm{sec}$. The primers used are listed in Table I.

Bioinformatics analysis. The target genes of selective exosomal miRNAs were predicted using TargetScan (http://www. targetscan.org/mamm_31/), miRDB (http://www.mirdb.org/), miRanda (http://www.microrna.org/microrna/home.do) and StarBase (http://starBase.sysu.edu.cn/). Only target genes that were found by 3 of the 4 tools were identified to be the target genes of exosomal miRNAs. The comprehensive function annotations of the potential targets of selective exosomal miRNAs were performed with Gene Ontology (GO) and Kyoto Encyclopedia of Genes and Genomes (KEGG) analysis based on the DAVID 6.7 software (http://david.abcc.ncifcrf. gov/home.jsp). All GO terms and signaling pathways were analyzed with a threshold of significance that was defined by a P-value of $<0.05$.

\section{Results}

Isolation and characterization of exosomes derived from the LSCC cell line AMC-HN-8. Our first step was to capture and verify the exosomes derived from LSCC cell line AMC-HN-8. Exosomes were isolated from conditioned medium of AMC-HN-8 using Ribo ${ }^{\mathrm{TM}}$ Exosome Isolation Reagent. After isolation, the morphology of exosome pellets was examined by TEM revealing membrane-bound particles that were homogeneous in appearance (Fig. 1A). In addition, the size distribution of exosome pellets was analyzed using NTA and the results revealed prospective diameters which
Table I. The sequences of primers.

Sequence

name

Primer sequences

miR-93-5p Forward: 5'GGCAAAGTGCTGTTCGTG3' Reverse: 5'CAGTGCGTGTCGTGGAGT3'

miR-1246 Forward: 5'GGGGAATGGATTTTTGG3' Reverse: 5'CAGTGCGTGTCGTGGAG3'

miR-122-5p Forward: 5'GGGTGGAGTGTGACAATGG3' Reverse: 5'CAGTGCGTGTCGTGGAGT3'

miR-320d Forward: 5'AAAAGCTGGGTTGAGAGGA3' Reverse: 5'CAGTGCGTGTCGTGGAGT3'

miR-4483 Forward: 5'GGGAAAAAGGGGTGGTCTG3' Reverse: 5'GTGCGTGTCGTGGAGTCG3'

miR-30c-5p Forward: 5'GGGGGTGTAAACATCCTAC3' Reverse: 5'GTGCGTGTCGTGGAGTCG3'

let-7e-5p Forward:5'GGGGTGAGGTAGGAGGTTGT3' Reverse: 5'GTGCGTGTCGTGGAGTCG3'

ranged from 30-150 nm (Fig. 1B). Furthermore, we assessed two well-established surface markers for exosomes, CD63 and CD81, using flow cytometry (Fig. 1C). The results revealed a significant difference after incubation with CD63 and CD81 antibodies. These results indicated that these pellets which we isolated had the size $(30-150 \mathrm{~nm})$, specific surface markers and morphology typical of that of exosomes.

MicroRNA expression profiles are significantly altered between parental cells and exosomes. To identify the miRNA expression profiles of parental cells and exosomes to investigate which miRNAs are selectively enclosed in exosomes, we performed small RNA sequencing. First, total RNA was extracted from parental cells $(n=3)$ and exosomes $(n=3)$. A small RNA library preparation and sample sequencing were conducted under the assistance of Guangzhou RiboBio Co., Ltd. The miRNAs were the most common among the known sequences, followed by tRNAs, snRNAs, Y RNAs, piRNAs and other RNAs which can not be classified. Of all the RNA examined, an average of $72.99 \%$ miRNAs were detected in parental cells compared with an average of $69.76 \%$ miRNAs in exosomes derived from parental cells, which did not exhibit an obvious discrepancy in composition of small RNAs (Fig. 2A). However, only $5.63 \%$ miRNAs were common for both cellular miRNAs and exosomal miRNAs, which meant that the process of miRNA packing into exosomes is selective (Fig. 2B). The most abundant miRNA in cells and exosomes was miR-21-5p and miR-1246, respectively (Fig. 2C). miR-21-5p has been previously reported to act as a predictor of recurrence and novel biomarker of urothelial (20) and gastric carcinoma (21). In addition, exosomal miR-1246 induced cell motility and invasion in oral squamous cell carcinoma (22), and functioned as a promising biomarker in peripheral circulation of patients who have breast and colon cancer $(23,24)$. To explore the functions of selective miRNAs in exosomes, we 
A

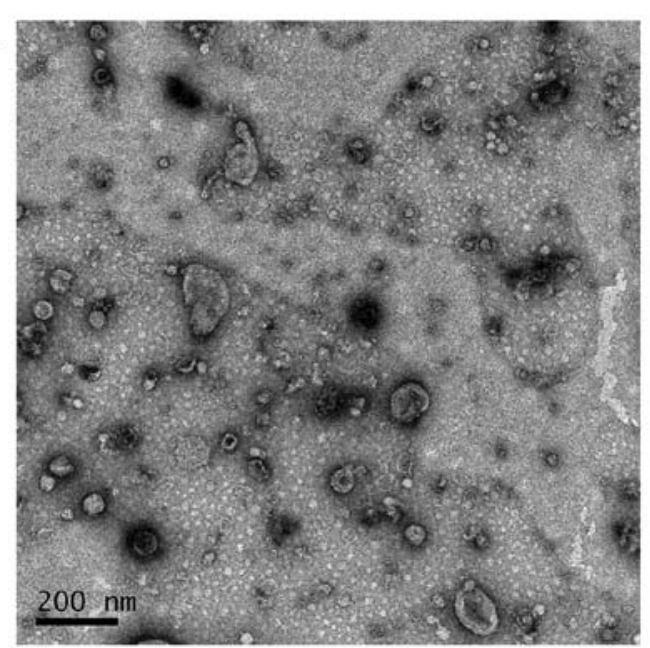

B

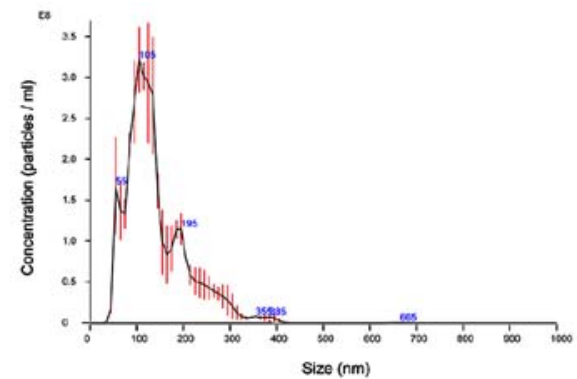

C

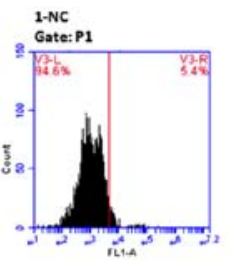

1-Co63

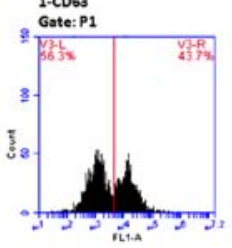

Figure 1. Characterization of exosomes. (A) Electron microscopy of exosomes derived from the LSCC cell line AMC-HN-8. The image revealed membrane-bound particles that were homogeneous in appearance and ranged from 30-150 nm in size. Scale bar, $200 \mathrm{~nm}$. (B) The diagram revealed the size distribution of exosomes by means of NTA. Error bars indicated +/-1 standard error of the mean. (C) Representative images of flow cytometry. Exosomes derived from the LSCC cell line AMC-HN-8 were stained with anti-CD63 and anti-CD81 immunofluorescence antibodies and analyzed by flow cytometry. Values indicate the ratio of positive exosomes. LSCC, laryngeal squamous cell carcinoma; NTA, nanoparticle tracking analysis.

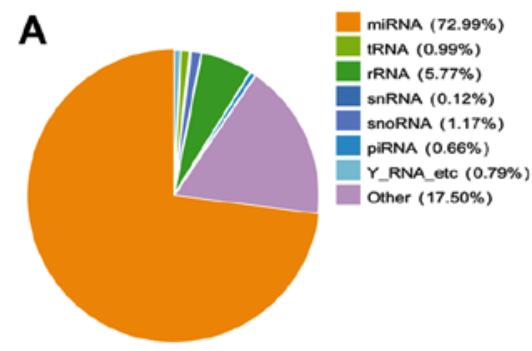

Total reads annotation in cells

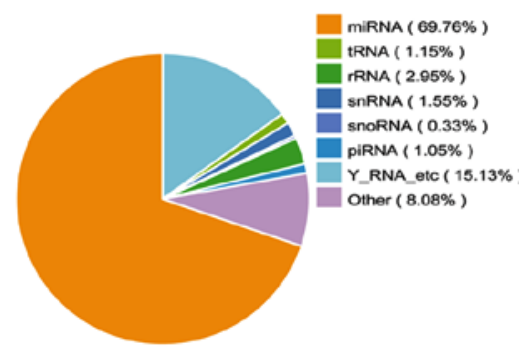

Total reads annotation in exosomes

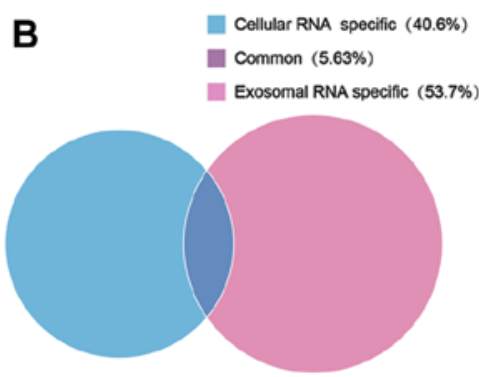

Top 10 miRnAs in exosomes
C

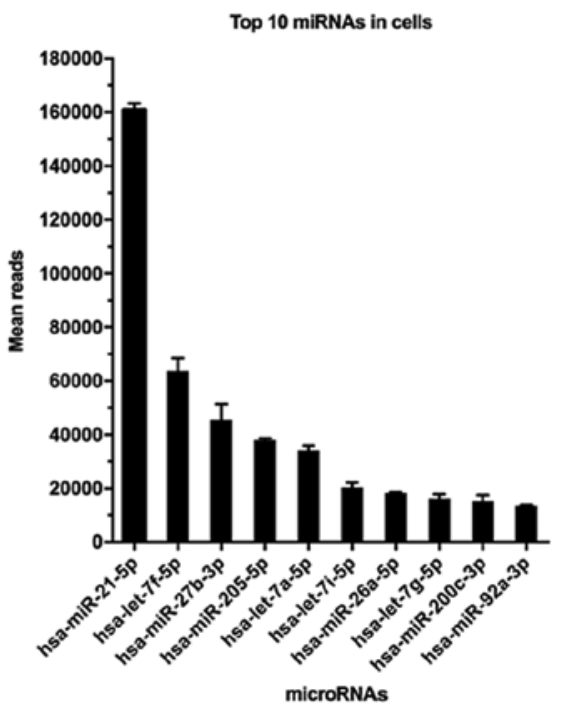

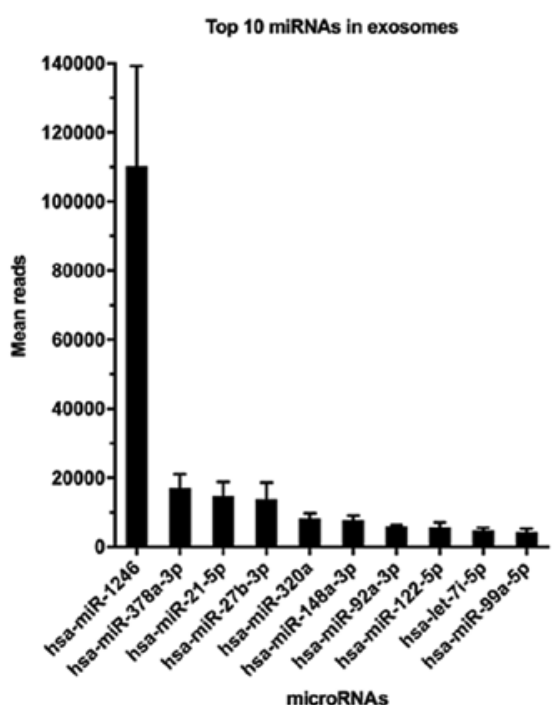

microRNAs

Figure 2. Comparison of total reads annotation in the cells and exosomes derived from LSCC cell line AMC-HN-8. (A) The total RNA read sequences in the cells and exosomes, revealed the annotation of ncRNA. The percentage is calculated as a percentage of clean reads. (B) The common reads of total RNA for cells and exosomes only accounted for 5.63\%. (C) The diagram displayed the top 10 miRNAs in cells and exosomes, respectively. LSCC, laryngeal squamous cell carcinoma.

then detected 15 miRNAs downregulated in exosomes and 14 miRNAs upregulated in exosomes as compared to parental cells, with both a P-value of $\leq 0.05$ and $>5$-fold-changes after filtering (Fig. 3). Detailed data are listed in Table II.
All of these data revealed altered miRNA expression profiles between parental cells and exosomes and indicated that miRNAs are selectively enriched or encapsulated in exosome pellets secreted from AMC-HN-8 cells rather than 
Table II. Expression of miRNAs in LSCC cell line AMC-HN-8 and in exosomes as compared to parental cells.

\begin{tabular}{|c|c|c|c|c|}
\hline miRNA & Mean reads in cells & Mean reads in exosomes & $\log _{2}$ (fold-change) & Upregulated/downregulated \\
\hline miR-1246 & 37.75 & 110304.50 & 11.43 & Up \\
\hline miR-1290 & 0.19 & 205.06 & 10.57 & Up \\
\hline miR-335-5p & 0.04 & 22.78 & 9.43 & Up \\
\hline miR-127-3p & 0.35 & 191.25 & 9.04 & Up \\
\hline miR-122-5p & 26.24 & 5701.51 & 7.91 & Up \\
\hline $\operatorname{miR}-369-3 p$ & 0.33 & 48.14 & 7.17 & Up \\
\hline miR-382-5p & 0.18 & 21.11 & 7.07 & Up \\
\hline miR-409-3p & 1.35 & 80.08 & 5.89 & Up \\
\hline miR-543 & 3.44 & 142.28 & 5.60 & Up \\
\hline miR-495-3p & 0.82 & 32.45 & 5.31 & Up \\
\hline $\operatorname{miR}-320 \mathrm{~d}$ & 22.95 & 967.48 & 5.31 & Up \\
\hline $\operatorname{miR}-320 \mathrm{e}$ & 1.12 & 46.15 & 5.25 & Up \\
\hline miR-345-3p & 1.26 & 32.17 & 5.01 & Up \\
\hline miR-143-3p & 6.70 & 130.52 & 5.01 & Up \\
\hline $\operatorname{miR}-27 a-5 p$ & 699.66 & 15.40 & -5.57 & Down \\
\hline miR-339-5p & 230.24 & 5.14 & -5.71 & Down \\
\hline miR-96-5p & 988.10 & 14.73 & -6.05 & Down \\
\hline let-7e-5p & 1109.58 & 15.48 & -6.26 & Down \\
\hline miR-23b-3p & 3611.89 & 51.95 & -6.26 & Down \\
\hline miR-135b-5p & 401.53 & 5.11 & -6.28 & Down \\
\hline $\operatorname{miR}-374 a-5 p$ & 500.32 & 3.85 & -7.10 & Down \\
\hline miR-30c-5p & 4737.26 & 34.67 & -7.17 & Down \\
\hline $\operatorname{miR}-374 a-3 p$ & 259.11 & 1.50 & -7.49 & Down \\
\hline miR-454-3p & 148.35 & 0.80 & -7.59 & Down \\
\hline $\operatorname{miR}-374 b-5 p$ & 639.38 & 3.05 & -7.82 & Down \\
\hline miR-29b-3p & 308.53 & 0.98 & -8.08 & Down \\
\hline miR-30b-5p & 831.74 & 2.22 & -8.57 & Down \\
\hline $\operatorname{miR}-4483$ & 948.75 & 0.87 & -9.96 & Down \\
\hline miR-4521 & 680.15 & 0.10 & -14.45 & Down \\
\hline
\end{tabular}

Upregulated/downregulated, in the exosomes as compared to the parental cells. Only miRNAs which exhibited the same trend in expression are listed. LSCC, laryngeal squamous cell carcinoma.

indiscriminate. These selective miRNAs in exosomes may promote malignant biological properties of tumors and play a crucial role in the cell-cell communication, including tumor cells-tumor cells or tumor cells-stromal cells crosstalk.

$R T-q P C R$ reveals the same trend as the sequencing results. To validate the results of small RNA sequencing, RT-qPCR was carried out to detect 3 upregulated miRNAs (miR-320d, miR-1246 and miR-122-5p) and 3 downregulated miRNAs (miR-4483, miR-30c-5p and let-7e-5p) in exosomes derived from 3 cell lines. Since no internal controls for exosomal microRNA analysis have been established, we used miR-93-5p due to its stable expression level between cells and exosomes. As anticipated, the results revealed that the expression level of miR-320d, miR-1246 and miR-122-5p was significantly upregulated, and miR-4483, miR-30c-5p and let-7e-5p was significantly downregulated in exosomes derived from all 3 cell lines, respectively (Fig. 4). These results were consistent with the small RNA sequencing data, which signified that the results of the small RNA sequencing were credible.

GO and KEGG pathway enrichment analysis of selective exosomal miRNAs. Furthermore, GO annotation and KEGG pathway enrichment analysis were performed to explore the potential targets of these 29 selective exosomal miRNAs. The results revealed that a total of $485 \mathrm{GO}$ terms were involved in biological processes, with $\mathrm{P}<0.05$ (Fig. 5A). The top 3 biological processes with the lowest P-value were related to vasculature development, regulation of transcription from RNA polymerase II promoter and negative regulation of macromolecule biosynthetic process. Up to $51 \mathrm{GO}$ terms were in relation to cellular components, with $\mathrm{P}<0.05$. The top 


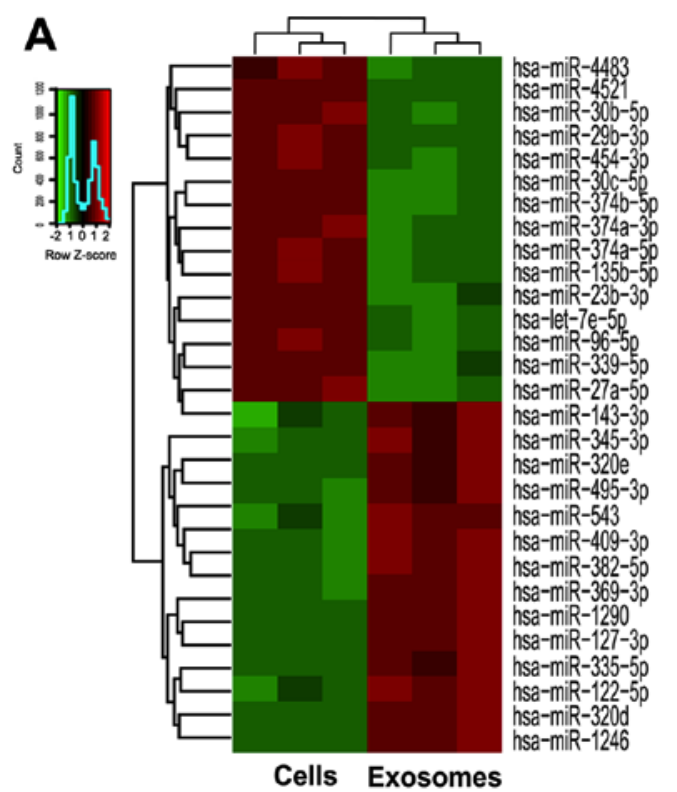

B

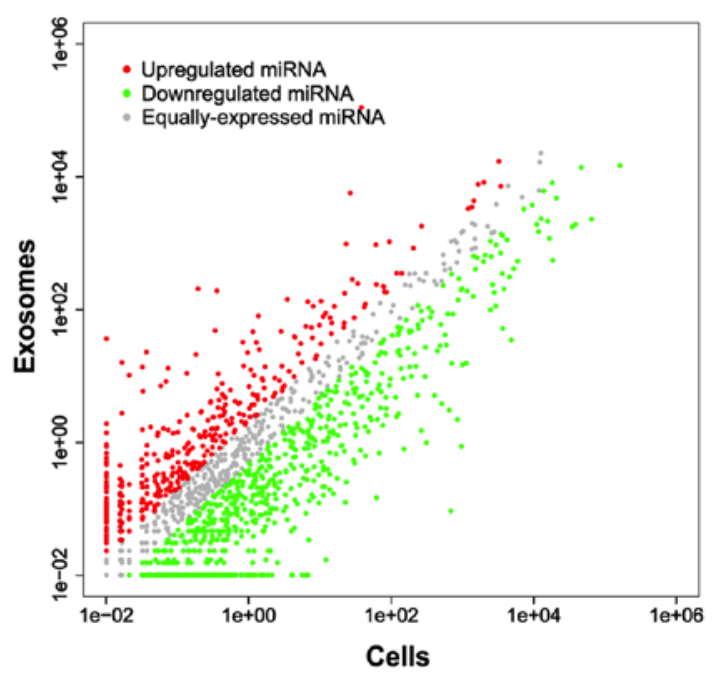

Figure 3. The different microRNA expression profiles between parental cells and exosomes. (A) The heat map revealed the distinct miRNA expression profiles between parental cells and exosomes. The inclusion criteria was a 2 -fold difference of $\log _{2}$ (fold-change) in either direction with a P-value $<0.05$. Red signal, high relative expression; green signal, low relative expression. (B) The volcano plot revealed the marked expression of miRNAs in exosomes. The inclusion criteria was a 2 -fold difference of $\log _{2}$ (fold-change) in either direction with a P-value $<0.05$. Red, upregulated in the exosomes; green, downregulated in the exosomes.
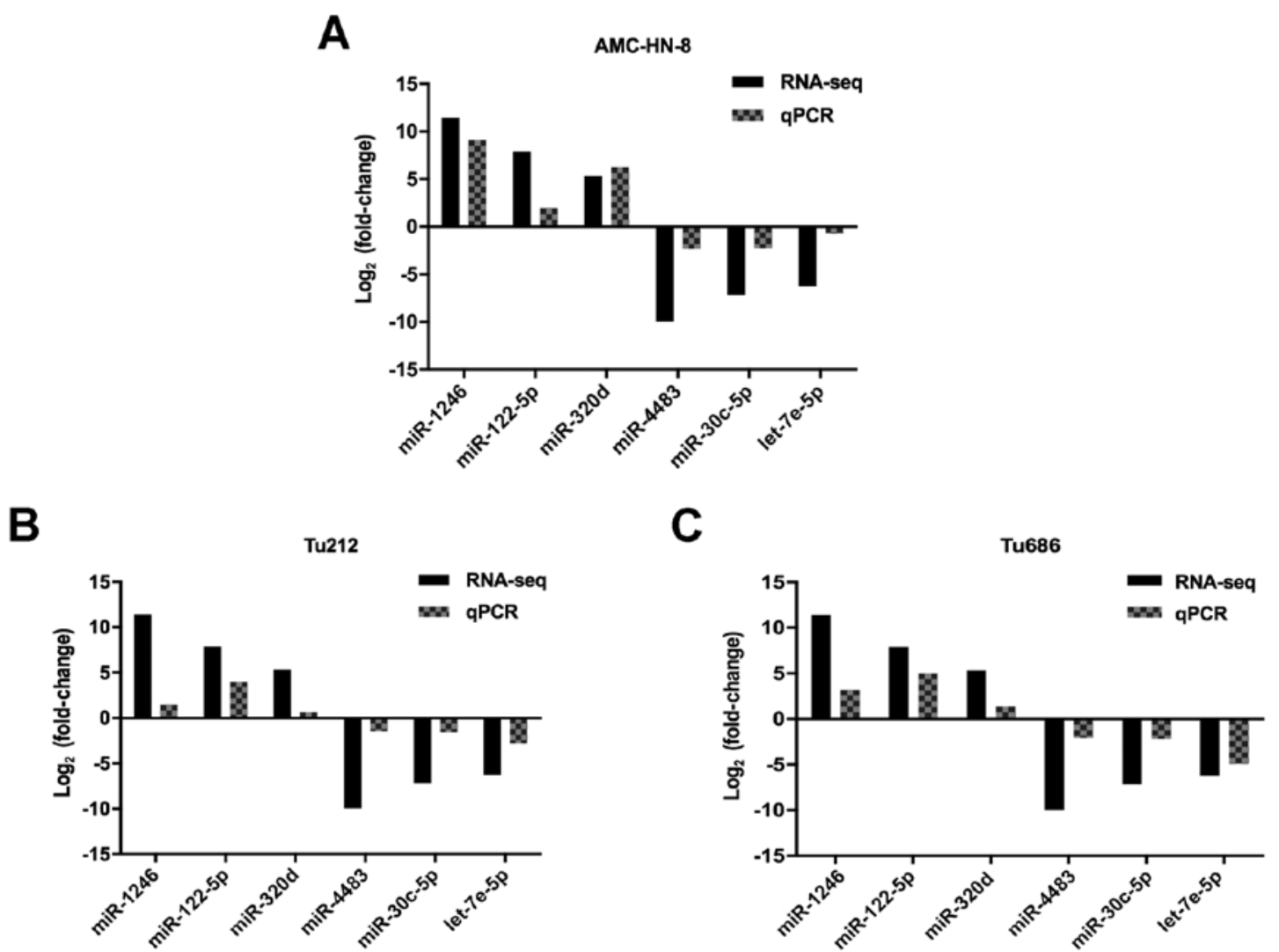

Figure 4. RT-qPCR validation. RT-qPCR was used to validate the results of small RNA sequencing. Six miRNAs accepted in the analysis (miR-320d, miR-1246, miR-122-5p, miR-4483, miR-30c-5p and let-7e-5p) were selected randomly. (A) LSCC cell line AMC-HN-8. (B) LSCC cell line Tu212. (C) LSCC cell line Tu686. The obtained values were normalized to hsa-miR-93-5p used as an internal control. Black, RNA-sequencing; Grey, RT-qPCR. RT-qPCR, reverse transcription quantitative PCR; LSCC, laryngeal squamous cell carcinoma.

3 cellular components with the lowest P-value were related to nucleoplasm, complex of collagen trimers and extracellular matrix component. There were $88 \mathrm{GO}$ terms related to molecular function in all, with $\mathrm{P}<0.05$. The top 3 molecular 
A

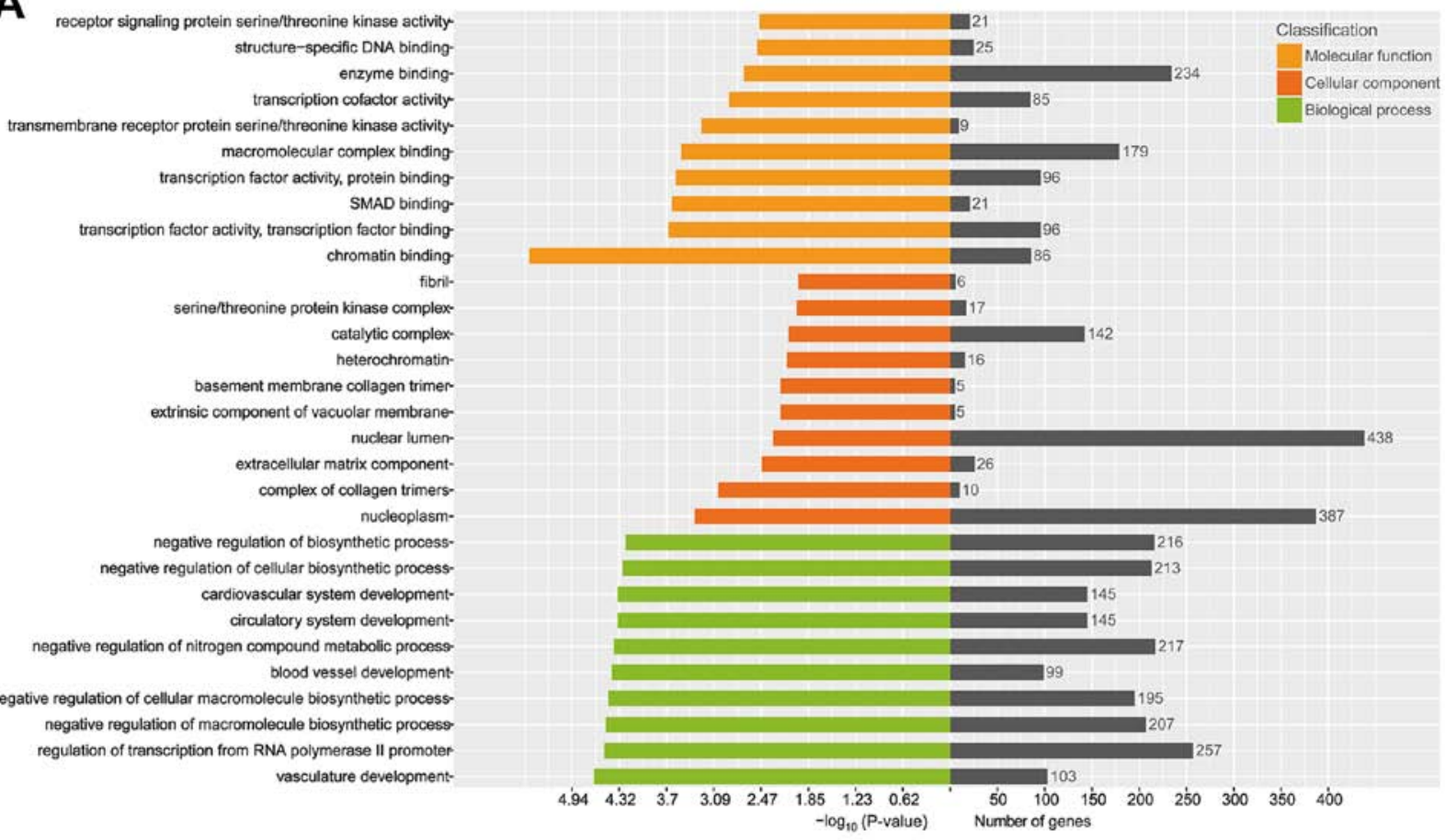

B

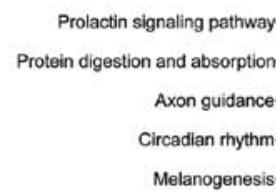

AGE-RAGE signaling pathway in diabetic complications-

Small cell lung cancer-

EGFR tyrosine kinase inhibitor resistance-

Renal cell carcinoma-

Pathways in cancer-

Hepatitis C-

Colorectal cancer-

Pancreatic cancer-

Amoebiasis-

Toxoplasmosis-

Chagas disease (American trypanosomiasis)-

mTOR signaling pathway-

HIF-1 signaling pathway-

PI3K-Akt signaling pathway-

FoxO signaling pathway-

TGF-beta signaling pathway-

p53 signaling pathway-

Focal adhesion-

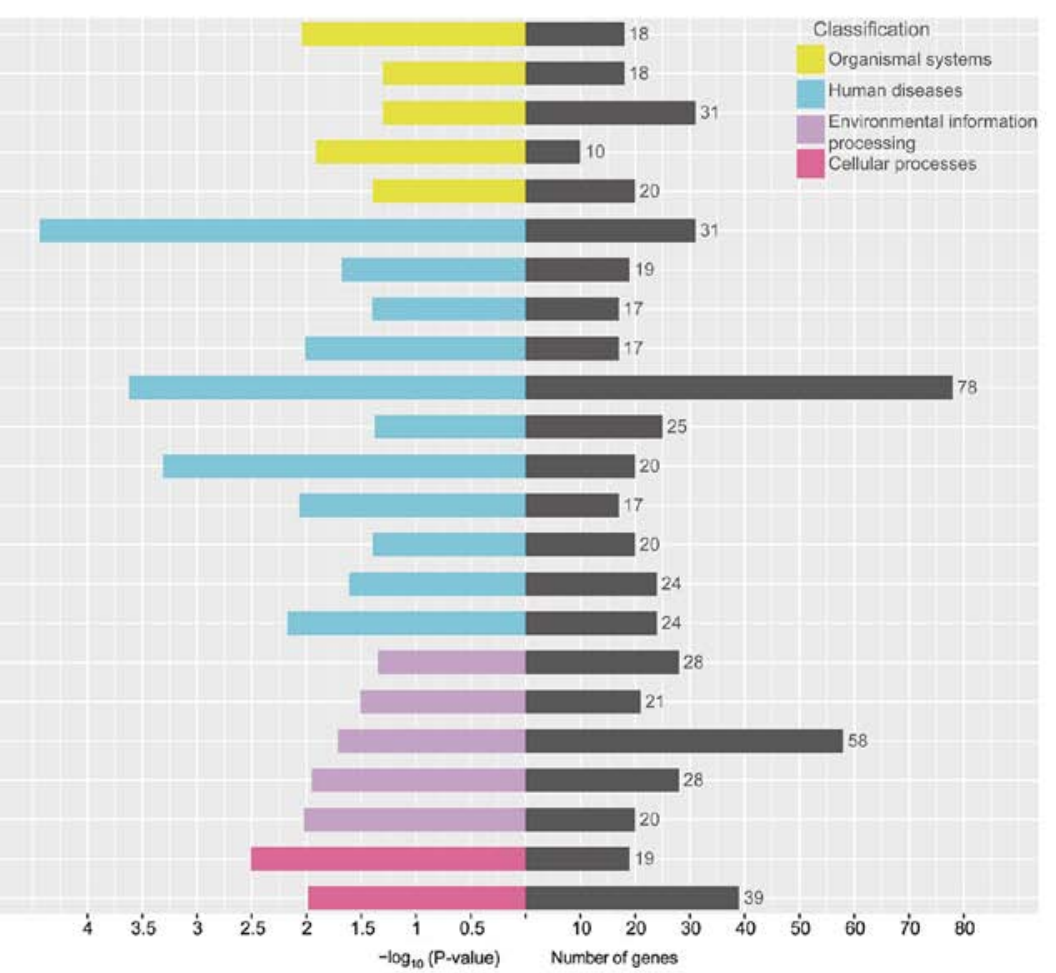

Figure 5. Bioinformatics analysis of potential targets of selective exosomal miRNAs. (A) GO annotation of predicted targets. (B) KEGG pathway enrichment analysis of predicted targets. GO terms and KEGG pathway both with a P-value <0.05 were selected. GO, Gene Ontology; KEGG, Kyoto Encyclopedia of Genes and Genomes.

functions with the lowest P-value were related to chromatin binding, transcription factor activity, transcription factor binding and SMAD binding.

The KEGG pathway enrichment analysis indicated that 279 pathways were involved in the current small RNA sequencing data, in which many of these pathways were related to organismal systems, cellular processes, environmental information processing and human diseases (Fig. 5B).
The top 5 pathways, with the lowest P-value, were the AGE-RAGE signaling pathway in diabetic complications, pathways in cancer, colorectal cancer, the p53 signaling pathway and signaling pathways regulating pluripotency of stem cells.

These results indicated that selective exosomal miRNAs play a distinctly important role in the process of cell metabolism, cell cycle and tumorigenesis. 

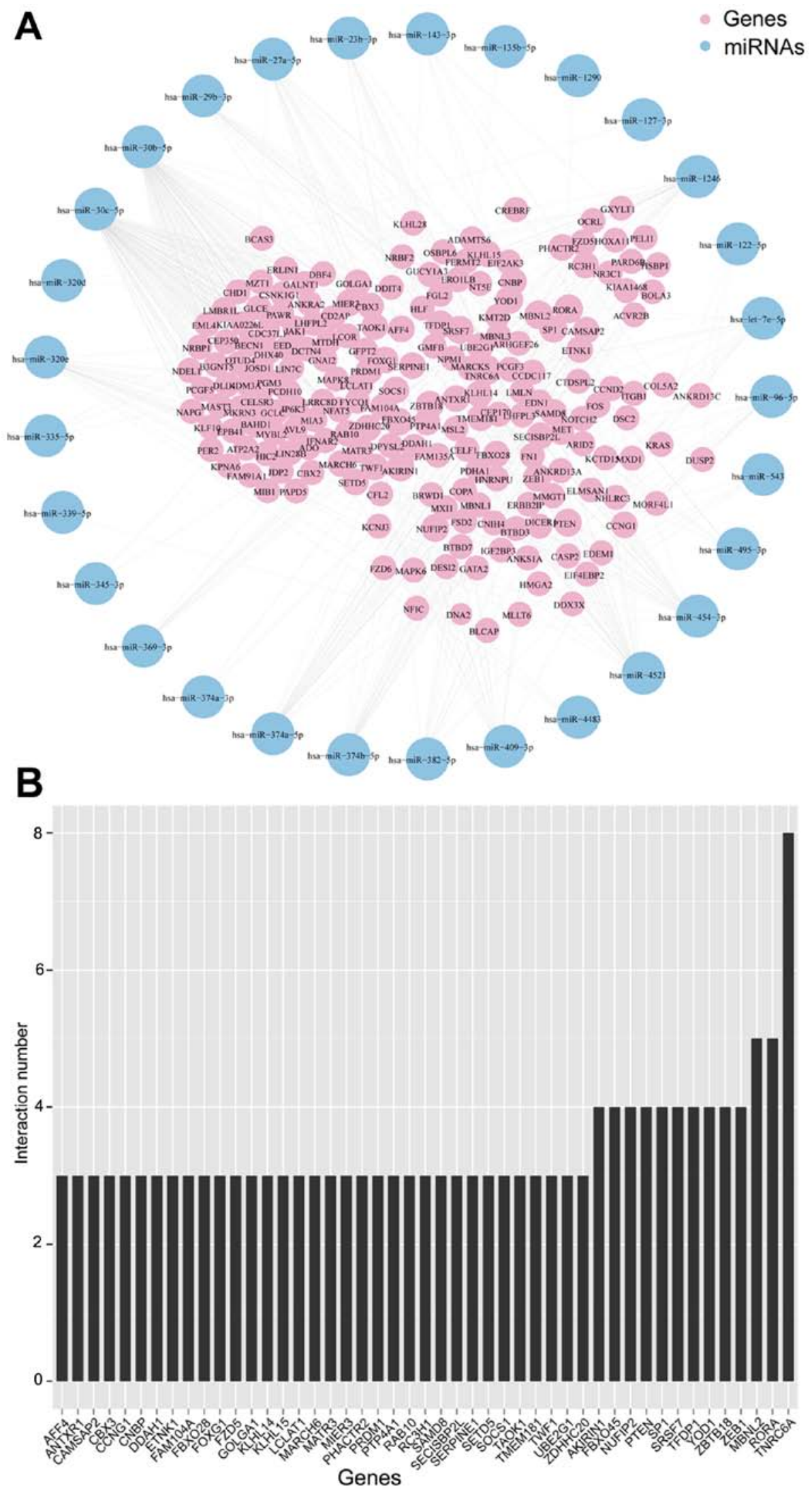

Figure 6. Selective exosomal miRNA-gene co-expression network. (A) The network revealed the interactions between exosomal miRNAs and genes. Blue, miRNAs; Pink, genes. (B) The most common overlap of genes.

Selective exosomal miRNA-gene co-expression network. We then constructed the co-expression network of selective exosomal miRNAs and genes (Fig. 6A). The most common overlay was the gene 'TNRC6A' (Fig. 6B), which was previously reported that along with Ago2, was overexpressed in the tissue of prostate and esophageal cancers (25). This network 
indicated that one single miRNA was bound up with a couple of genes and vice versa.

\section{Discussion}

Exosomes are nanometer-scale membrane vesicles (ranging from 30-150 nm) of endocytic origin which are released into the extracellular environment. Since exosomes are found in the supernatant of maturing sheep reticulocytes $(26,27)$, studies have increasingly demonstrated that almost all types of mammalian cells can release exosomes (8) and exosomes act as messengers carrying bioinformation in the process of cell-cell communication rather than a 'waste bin' for excreting useless content (28). MicroRNAs (miRNAs) are short (18-24 nt) ncRNAs that are associated with post-transcriptional regulation of gene expression by affecting both the stability and translation of mRNAs (29). Exosomal miRNAs have gained much attention since Valadi et al (10) demonstrated that exosomes contain miRNAs and these so-called 'exosomal miRNAs' can be delivered into the extracellular environment, taken up by adjacent cells or carried to distant sites via biological fluids. These exosomal miRNAs may therefore induce the phenotypic modifications in recipient cells, indicating that exosomes are important mediators of intercellular communication and may play a significant role on the malignant process of tumors.

Previous studies $(30,31)$ demonstrated that some specific miRNAs may be excreted or retained in the cells preferentially, which means the process by which several miRNAs are enclosed in exosomes is selective rather than indiscriminate. These results implied that these selective exosomal miRNAs may play a significant role in tumor malignant behaviors. Therefore, we conducted small RNA sequencing to detect the difference between LSCC AMC-HN-8 cells and exosomes which were secreted from this cell line.

The LSCC cell line AMC-HN-8 was cultured in the conditioned medium containing 10\% exosome-depleted FBS (excluding the contamination from bovine exosomes) for $72 \mathrm{~h}$ at $90-100 \%$ density. After preprocessing the conditioned medium by centrifugation, filtration and concentration, exosomes were isolated with Ribo ${ }^{\mathrm{TM}}$ Exosome Isolation Reagent (32). To characterize the exosomes pellets, we performed TEM and NTA that are universally recognized (33), and membrane-bound particles that were homogeneous in appearance and ranging from $30-150 \mathrm{~nm}$ in size were observed. Additionally, we detected two well-established exosome surface markers CD63 and CD81 with flow cytometry. After staining with the specific antibody, the positive rate ranged from 43.7-75.9\%, which exhibited a significant difference. Specific combining capacity of antibodies and distribution of antigens may contribute to the slight difference of a positive rate of two antibodies in one sample. Now that we successfully isolated the exosome pellets derived from LSCC, small RNA sequencing was performed.

Then, we identified the miRNA expression profiles of parental cells and exosomes to investigate which miRNAs are selectively enclosed in exosomes via small RNA sequencing. As the most common known sequences in the cell, miRNAs occupied the largest portion which was $72.99 \%$ followed by snRNAs, snoRNAs, piRNAs and Y RNAs (Fig. 2A), which acknowledged that miRNAs were the pivotal mediators in the vital movement. Similar with the content of cells, exosomal
miRNAs derived from AMC-HN-8 cells made up to $69.76 \%$ of whole small RNAs. However, only 5.63\% miRNAs were detected commonly both in cells and exosomes and 53.7\% miRNAs were exclusively detected in exosomes suggesting a very notable enrichment in exosomes compared to parental cells (14). According to the results, miR-21, let-7f-5p and miR-27-3p were identified as the 3 most abundant miRNA types in cells, and these miRNAs had been previously reported to take part in tumor development, including gastric (21), laryngeal (34) and esophageal carcinoma (35). Largely different from cellular miRNAs, miR-1246 ranked first among exosomes which was consistent with previous research $(22,23,36)$ indicating that exosomal miR-1246 may take part in tumor development regardless of the type of tumor. Subsequently, miR-378a-3p ranked second among exosomes, and miR-378a-3p was reported to also exist in the exosomes derived from HPV-positive cervical carcinoma cells (17). miR-21-5p, consistent with cellular content, was also enriched in exosomes and ranked third.

Subsequently, we investigated miRNAs with $\log _{2}$ (foldchange) $>2$ and revealed their expression level with heat map and volcano plots (Fig. 3). We identified miRNAs that were enriched in cells or exosomes. To further narrow the research scope, we selected 15 miRNAs that were downregulated in exosomes and 14 miRNAs that were upregulated in exosomes as compared to parental cells, with a P-value of both $\leq 0.05$ and $>5$-fold-changes. These miRNAs are listed in Table II. To validate the results of sequencing, RT-qPCR was performed to detect 6 randomly selected miRNAs (miR-320d, miR-1246, miR-122-5p, miR-4483, miR-30c-5p and let-7e-5p) in 3 cell lines (AMC-HN-8, Tu212 and Tu686). From the results obtained, we concluded that the qPCR results were in agreement with the sequencing results, and that the sequencing results were reliable.

Next, we predicted the potential targets of selective exosomal miRNAs through TargetScan, miRDB, miRanda and StarBase databases. Then, the comprehensive function annotations of the potential targets were performed with GO and KEGG pathway enrichment analysis. The GO analysis results revealed that the potential targets of selective exosomal miRNAs were involved in biological processes, cellular components and molecular functions, including vasculature development, nucleoplasm and chromatin binding. KEGG pathway enrichment analysis revealed that the AGE-RAGE signaling pathway and p53 signaling pathway may be involved in the process and development of these selective exosomal miRNAs.

We then constructed the co-expression network of selective exosomal miRNAs and genes (Fig. 5). In this network, we acquired specific information about the interrelation between these selective exosomal miRNAs and genes.

In conclusion, we successfully isolated the exosomes derived from LSCC cell line AMC-HN-8, and characterized the exosomes with TEM, NTA and flow cytometry. Through the assistance of small RNA sequencing combined with RT-qPCR analysis, we revealed a significant difference between cellular miRNAs and exosomal miRNAs, and that there is a very notable enrichment in exosomes compared to parental cells. After GO annotation and KEGG pathway enrichment analysis, we suggest that these selective exosomal 
miRNAs may contribute to tumor development from various perspectives.

\section{Acknowledgements}

Not applicable.

\section{Funding}

The present study was supported by the Science and Technology Commission of Shanghai Municipality, China (grant nos. 12J1402100 and 16411950101), and Shanghai ShenKang Hospital Development Center, China (grant no. SHDC12015114). The sponsors or funding organizations had no role in the design or execution of this research.

\section{Availability of data and materials}

The datasets used during the present study are available from the corresponding author upon reasonable request.

\section{Authors' contributions}

$\mathrm{QH}$ and LZ conceived and designed the study. QH, JY, JZ, $\mathrm{CH}$ and $\mathrm{YG}$ performed the experiments. $\mathrm{QH}$ and $\mathrm{LZ}$ wrote the paper. QH, LZ, JY, JZ, CH and YG reviewed and edited the manuscript. All authors read and approved the manuscript and agree to be accountable for all aspects of the research in ensuring that the accuracy or integrity of any part of the work are appropriately investigated and resolved.

\section{Ethics approval and consent to participate}

Not applicable.

\section{Patient consent for publication}

Not applicable.

\section{Competing interests}

The authors state that they have no competing interests.

\section{References}

1. Steuer CE, El-Deiry M, Parks JR, Higgins KA and Saba NF: An update on larynx cancer. CA Cancer J Clin 67: 31-50, 2017.

2. Siegel RL, Miller KD and Jemal A: Cancer statistics, 2016. CA Cancer J Clin 66: 7-30, 2016.

3. Foekens JA, Sieuwerts AM, Smid M, Look MP, de Weerd V, Boersma AW, Klijn JG, Wiemer EA and Martens JW: Four miRNAs associated with aggressiveness of lymph node-negative, estrogen receptor-positive human breast cancer. Proc Natl Acad Sci USA 105: 13021-13026, 2008.

4. Kelly EJ, Hadac EM, Greiner S and Russell SJ: Engineering microRNA responsiveness to decrease virus pathogenicity. Nat Med 14: 1278-1283, 2008.

5. Ji J, Shi J, Budhu A, Yu Z, Forgues M, Roessler S, Ambs S, Chen Y, Meltzer PS, Croce CM, et al: MicroRNA expression, survival, and response to interferon in liver cancer. N Engl J Med 361: 1437-1447, 2009.

6. Cao P, Zhou L, Zhang J, Zheng F, Wang H, Ma D and Tian J: Comprehensive expression profiling of microRNAs in laryngeal squamous cell carcinoma. Head Neck 35: 720-728, 2013.
7. Hui AB, Lenarduzzi M, Krushel T, Waldron L, Pintilie M, Shi W, Perez-Ordonez B, Jurisica I, O'Sullivan B, Waldron J, et al: Comprehensive MicroRNA profiling for head and neck squamous cell carcinomas. Clin Cancer Res 16: 1129-1139, 2010.

8. Tkach M and Théry C: Communication by extracellular vesicles: Where we are and where we need to go. Cell 164: 1226-1232, 2016.

9. Van Niel G, D'Angelo G and Raposo G: Shedding light on the cell biology of extracellular vesicles. Nat Rev Mol Cell Biol 19: 213-228, 2018.

10. Valadi H, Ekström K, Bossios A, Sjöstrand M, Lee JJ and Lötvall JO: Exosome-mediated transfer of mRNAs and microRNAs is a novel mechanism of genetic exchange between cells. Nat Cell Biol 9: 654-659, 2007.

11. Ye SB, Zhang H, Cai TT, Liu YN, Ni JJ, He J, Peng JY, Chen QY, Mo HY, Jun-Cui, et al: Exosomal miR-24-3p impedes T-cell function by targeting FGF11 and serves as a potential prognostic biomarker for nasopharyngeal carcinoma. J Pathol 240: 329-340, 2016.

12. Li L, Li C, Wang S, Wang Z, Jiang J, Wang W, Li X, Chen J, Liu K, Li C, et al: Exosomes derived from hypoxic oral squamous cell carcinoma cells deliver miR-21 to normoxic cells to elicit a prometastatic phenotype. Cancer Res 76: 1770-1780, 2016.

13. Wang J, Zhou Y, Lu J, Sun Y, Xiao H, Liu M and Tian L: Combined detection of serum exosomal miR-21 and HOTAIR as diagnostic and prognostic biomarkers for laryngeal squamous cell carcinoma. Med Oncol 31: 148, 2014

14. Kogure T, Lin WL, Yan IK, Braconi C and Patel T: Intercellular nanovesicle-mediated microRNA transfer: A mechanism of environmental modulation of hepatocellular cancer cell growth. Hepatology 54: 1237-1248, 2011.

15. Dickman CT, Lawson J, Jabalee J, MacLellan SA, LePard NE, Bennewith KL and Garnis C: Selective extracellular vesicle exclusion of miR-142-3p by oral cancer cells promotes both internal and extracellular malignant phenotypes. Oncotarget 8: 15252-15266, 2017.

16. Lawson J, Dickman C, MacLellan S, Towle R, Jabalee J, Lam S and Garnis C: Selective secretion of microRNAs from lung cancer cells via extracellular vesicles promotes CAMK1D-mediated tube formation in endothelial cells. Oncotarget 8: 83913-83924, 2017.

17. Honegger A, Schilling D, Bastian S, Sponagel J, Kuryshev V, Sültmann H, Scheffner M, Hoppe-Seyler K and Hoppe-Seyler F: Dependence of intracellular and exosomal microRNAs on viral E6/E7 oncogene expression in HPV-positive tumor cells. PLoS Pathog 11: e1004712, 2015.

18. Kim SY, Chu KC, Lee HR, Lee KS and Carey TE: Establishment and characterization of nine new head and neck cancer cell lines. Acta Otolaryngol 117: 775-784, 1997.

19. Livak KJ and Schmittgen TD: Analysis of relative gene expression data using real-time quantitative PCR and the $2^{-\Delta \Delta C_{\mathrm{T}}}$ method. Methods 25: 402-408, 2001.

20. Matsuzaki K, Fujita K, Jingushi K, Kawashima A, Ujike T, Nagahara A, Ueda Y, Tanigawa G, Yoshioka I, Ueda K, et al: MiR-21-5p in urinary extracellular vesicles is a novel biomarker of urothelial carcinoma. Oncotarget 8: 24668-24678, 2017.

21. Park SK, Park YS, Ahn JY, Do EJ, Kim D, Kim JE, Jung K, Byeon JS, Ye BD, Yang DH, et al: MiR 21-5p as a predictor of recurrence in young gastric cancer patients. J Gastroenterol Hepatol 31: 1429-1435, 2016.

22. Sakha S, Muramatsu T, Ueda $\mathrm{K}$ and Inazawa J: Exosomal microRNA miR-1246 induces cell motility and invasion through the regulation of DENND2D in oral squamous cell carcinoma. Sci Rep 6: 38750, 2016.

23. Hannafon BN, Trigoso YD, Calloway CL, Zhao YD, Lum DH, Welm AL, Zhao ZJ, Blick KE, Dooley WC and Ding WQ: Plasma exosome microRNAs are indicative of breast cancer. Breast Cancer Res 18: 90, 2016.

24. Ogata-Kawata H, Izumiya M, Kurioka D, Honma Y, Yamada Y, Furuta K, Gunji T, Ohta H, Okamoto H, Sonoda H, et al: Circulating exosomal microRNAs as biomarkers of colon cancer. PLoS One 9: e92921, 2014.

25. Yoo NJ, Hur SY, Kim MS, Lee JY and Lee SH: Immunohistochemical analysis of RNA-induced silencing complex-related proteins AGO2 and TNRC6A in prostate and esophageal cancers. APMIS 118: 271-276, 2010.

26. Pan BT, Teng K, Wu C, Adam M and Johnstone RM: Electron microscopic evidence for externalization of the transferrin receptor in vesicular form in sheep reticulocytes. J Cell Biol 101: 942-948, 1985. 
27. Johnstone RM, Adam M, Hammond JR, Orr L and Turbide C: Vesicle formation during reticulocyte maturation. Association of plasma membrane activities with released vesicles (exosomes). J Biol Chem 262: 9412-9420, 1987.

28. Becker A, Thakur BK, Weiss JM, Kim HS, Peinado H and Lyden D: Extracellular vesicles in cancer: Cell-to-Cell mediators of metastasis. Cancer Cell 30: 836-848, 2016.

29. Guo H, Ingolia NT, Weissman JS and Bartel DP: Mammalian microRNAs predominantly act to decrease target mRNA levels. Nature 466: 835-840, 2010.

30. Baglio SR, Rooijers K, Koppers-Lalic D, Verweij FJ Pérez Lanzón M, Zini N, Naaijkens B, Perut F, Niessen HW Baldini N, et al: Human bone marrow- and adipose-mesenchymal stem cells secrete exosomes enriched in distinctive miRNA and tRNA species. Stem Cell Res Ther 6: 127, 2015.

31. Endzelinš E, Berger A, Melne V, Bajo-Santos C, Sobolevska K, Ābols 'A, Rodriguez M, Santare D, Rudnickiha A, Lietuvietis V, et al: Detection of circulating miRNAs: Comparative analysis of extracellular vesicle-incorporated miRNAs and cell-free miRNAs in whole plasma of prostate cancer patients. BMC Cancer 17: 730, 2017.

32. Chen J, Yu Y, Li S, Liu Y, Zhou S, Cao S, Yin J and Li G: MicroRNA-30a ameliorates hepatic fibrosis by inhibiting Beclin1-mediated autophagy. J Cell Mol Med 21: 3679-3692, 2017.
33. Théry C, Amigorena S, Raposo G and Clayton A: Isolation and characterization of exosomes from cell culture supernatants and biological fluids. Curr Protoc Cell Biol Chapter 3: Unit 3.22, 2006.

34. Yao XD, Li P and Wang JS: MicroRNA differential expression spectrum and microRNA-125a-5p inhibition of laryngeal cancer cell proliferation. Exp Ther Med 14: 1699-1705, 2017.

35. Hummel R, Sie C, Watson DI, Wang T, Ansar A, Michael MZ, Van der Hoek M, Haier J and Hussey DJ: MicroRNA signatures in chemotherapy resistant esophageal cancer cell lines. World J Gastroenterol 20: 14904-14912, 2014.

36. Xu YF, Hannafon BN, Zhao YD, Postier RG and Ding WQ: Plasma exosome miR-196a and miR-1246 are potential indicators of localized pancreatic cancer. Oncotarget 8: 77028-77040, 2017. Attribution-NonCommercial-NoDerivatives 4.0 International (CC BY-NC-ND 4.0) License. 